\title{
Global well-posedness for the generalized Navier-Stokes-Coriolis equations with highly oscillating initial data
}

\author{
Xiaochun Sun ${ }^{1}$, Mixiu Liu ${ }^{1}$, and Jihong Zhang ${ }^{2}$ \\ ${ }^{1}$ Northwest Normal University \\ ${ }^{2}$ Lanzhou City University
}

September 25, 2021

\begin{abstract}
We study the small initial date Cauchy problem for the generalized incompressible Navier-Stokes-Coriolis equations in critical hybrid-Besov space $\$ \backslash \operatorname{dot}\{\backslash \operatorname{mathscr}\{\mathrm{B}\}\}_{-}\{2, \backslash, \mathrm{p}\}^{\wedge}\{\backslash$ frac $\{5\}\{2\}-2 \backslash$ alpha, $\backslash$ frac $\{3\}\{\mathrm{p}\}-2 \backslash$ alpha +1$\}\left(\backslash \operatorname{mathbb}\{\mathrm{R}\}^{\wedge} 3\right) \$$ with $\$ 1 / 2<\backslash$ alpha $<2 \$$ and $\$ 2 \backslash$ leq $\mathrm{p} \backslash$ leq $4 \$$. We prove that hybrid-Besov spaces norm of a class of highly osillating initial velocity can be arbitrarily small. and we prove the estimation of highly frequency $\$ \mathrm{~L}^{\wedge} \mathrm{p} \$$ smoothing effect for generalized Stokes-Coriolis semigroup with $\$ 1 \backslash$ leq $\mathrm{p} \backslash$ leq $\backslash$ infty $\$$, At the same time, we prove space-time norm estimation of hybrid-Besov spaces for StokesCoriolis semigroup. From this result we deduce bilinear estimation in our work space. Our method relies upon Bony's high and low frequency decomposition technology and Banach fixed point theorem.
\end{abstract}

\section{Hosted file}

Globalwell-posednessforGNSCwithhighlyoscillatinginitialdata.pdf available at https: //authorea.com/users/330488/articles/538669-global-well-posedness-for-the-generalizednavier-stokes-coriolis-equations-with-highly-oscillating-initial-data

\section{Hosted file}

Globalwell-posednessforGNSCwithhighlyoscillatinginitialdata.tex available at https: //authorea.com/users/330488/articles/538669-global-well-posedness-for-the-generalizednavier-stokes-coriolis-equations-with-highly-oscillating-initial-data 